\title{
The clinical experience and potential of brain imaging in patients with mental illness
}

\author{
Belinda R. Lennox* \\ Department of Psychiatry, University of Cambridge, Cambridgeshire, UK \\ ${ }^{*}$ Correspondence: bl224@cam.ac.uk
}

\section{INTRODUCTION}

Magnetic resonance imaging (MRI) is rarely used as an investigation in clinical psychiatric practice. The reasons for this are several, and include the cultural viewpoint of psychiatry as a psychosocial specialty as well as particular concerns about the experience of brain imaging amongst patients and clinicians. Where MRI is used it is to exclude neurological disorders in those presenting with mental illness. However the yield of such disorders through MRI screening not detected through other clinical examination in 'typical' presentations of mental illness is very low, and routine MRI is not currently recommended for those with mental illness without warning signs of neurological disorder. However functional imaging does have clinical potential, with the possibility for assisting both with diagnosis and treatment planning, and this potential will be discussed.

\section{CULTURAL SHIFT IN THE VIEW OF PSYCHIATRY}

Psychiatry was originally described by Reil (1808) as a purely medical speciality that should be undertaken by only the best physicians, and into which philosophers and psychologists should not be incorporated. The specialty included the management of all disorders of the 'soul', including both mind and brain (Marneros, 2008).

Psychiatry as practiced today has evolved into a community based holistic specialty where psychosocial models of illness sit alongside biological perspectives, and patients are managed using a broad range of these approaches (Craddock et al., 2008). This practice has developed due to the lack of established 'organicity' of psychiatric illness and the prominence of psychological theorists in the 20th century. There is therefore currently an arbitrary division of brain disorders into the specialties of psychiatry and neurology, with different terminologies for the same phenomena, and different clinical practices. This has been to the detriment of patients on both sides of the divide. In practical terms this manifests itself with the ease of access to MRI on the neurological side, including an expectation from patients that this should be offered. The use of MRI is rare in psychiatric practice, however, both because of the psychosocial interpretation of symptoms amongst clinicians and patients, and the dispersed nature of the clinical practice. To incorporate functional magnetic resonance imaging (fMRI) scans into routine psychiatric practice, if this was shown to be useful, would therefore require a significant culture change. However there are clinical presentations that should include imaging as part of routine care. These include late onset first episode of mental illness (affective or psychotic), atypical presentations or where there is any other suspicion of neurological disorder (sensory or motor or cranial nerve disturbance, fits or loss of consciousness). The use of MRI and perfusion hexamethylpropyleneamine oxime (HMPAO) single-photon emission computed tomography (SPECT) in the early differential diagnosis of dementia sub-types should also be part of routine care. In these cases clinicians and patients should expect imaging to be part of the normal assessment protocol.

\section{CLINICAL EXPERIENCE}

A further reluctance of clinicians to refer patients for MRI scans may be a concern about the acceptability of the procedure to the patient - that the experience will be traumatic and may make people feel worse. MRI scanners are noisy and feel enclosed. The main cause of distress that people describe during MRI scans is claustrophobia. Certainly patients who are mentally ill and particularly if they have pathological levels of anxiety will have an increased chance of experiencing claustrophobia whilst in the scanner. Some patients who are already feeling vulnerable may feel traumatized by the experience. Furthermore, patients who are paranoid may become concerned that the scanner can read their mind and tell what they are thinking. The high tech nature of the investigation and the fact that patients are being observed and monitored from a separate room can be difficult to tolerate for patients who are acutely sensitive to other people's intentions towards them.

As well as being unpleasant for some patients because of the nature of mental illness, it may not be possible to achieve high quality images in acutely unwell patients because of movement. Patients who are manic in particular may have difficulty lying still. There would therefore have to be a high suspicion of pathology to persist with obtaining an MRI in an acutely mentally ill patient. There needs to be an individual judgement of possible benefit with the distress that may be caused in the patient.

\section{PATIENT EXPERIENCE}

In my experience there are both positive and negative views of brain imaging from patients with mental illness. The positive view is that brain imaging may be helpful in being able to validate the real nature of their experiences. So for instance, the ability to demonstrate auditory cortex activation during the subjective experience of auditory hallucinations shows in an objective way that patients really are hearing voices - the activation from the hallucination is of the same quality as the activation from external auditory stimuli (Lennox et al., 2001).

The negative view is that brain imaging is a biological and reductionist method of looking for abnormality in those with mental illness. Many people with mental illness object to having the individual nature of their experience reduced to an abnormal pattern of brain activation, or a structural deficit. The implication is that illness is predetermined by brain structure and function, and that there is little control the individual can have over their recovery. 
Mental illness is a complex interplay between both biological and environmental factors, and many interesting imaging studies are now showing both the effect of the environment on brain structure and function, and the plasticity of the brain, both to recover after injury and to increase in grey matter with training.

\section{CLINICAL POTENTIAL FOR BRAIN IMAGING}

Changes on structural MRI are seen in all the major psychiatric illnesses (schizophrenia, bipolar disorder, depression, obsessive compulsive disorder). However, these are not specific enough to warrant the routine use of structural MRI to diagnose these disorders. However functional brain imaging, currently a research tool, has the real prospect of helping in the diagnosis and treatment decisions for patients with mental illness in the near future. As an illustration I will focus on the use of AMRI in the diagnosis and treatment planning of those with depression.

\section{fMRI OF DEPRESSION}

Functional imaging relies on the change in blood oxygenation within the brain on performing a task, with the assumption that blood oxygenation is a proxy measure for brain activity. The task is used as a probe of areas relevant to the disorder being investigated. Hence in functional imaging of mood disorders, it is common to use emotional images, which activate areas important in processing emotion and then compare activation patterns with healthy controls. The areas of brain that increase in oxygenation as the images increase in intensity of emotion can be assumed to be the areas associated with processing of that emotion.

\section{fMRI TO PREDICT TREATMENT RESPONSE IN DEPRESSION}

Using these methods there are reliable patterns of abnormalities shown in the emotion processing network in depression, with underactivity in anterior cingulate, and overactivity of the amygdala in particular (Drevets et al., 1997). These areas are closely interconnected, with a feedback loop between the two, and the underactivity in cingulate causing consequent overactivity in amygdala. It appears as though while abnormality in both regions is required for the clinical syndrome, normalizing the abnormality in either region can result in treatment improvement. The level of amygdala overactivity has been shown to correlate with severity of illness as measured clinically, and is coupled with treatment response - normalising with effective treatment with antidepressants and persisting in those not responding to treatment (Chen et al., 2008). In contrast, psychological treatment has a more 'top-down' effect, altering prefrontal activation directly rather than the amygdalae (Fu et al., 2008).

Functional imaging may therefore be helpful in tailoring treatment for those with depression - those with the highest level of amygdala overactivation being most likely to benefit from antidepressants, and those with predominantly prefrontal underactivation being most likely to benefit from cognitive behavioural therapy.

\section{fMRI TO DIAGNOSE DEPRESSION}

Bipolar disorder and unipolar depression are separate disorders with distinct pharmacological treatments. In those presenting with depression without a previous history of mania it is not possible to distinguish the two disorders on clinical features alone. Up to $28 \%$ of those with depression will progress to having a diagnosis of bipolar disorder (Hantouche et al., 1998). Functional imaging studies indicate different patterns of brain activity in those with bipolar and unipolar depression (Savitz and Drevets, 2009). fMRI could therefore be of diagnostic use in those with depression.

\section{fMRI TO AID DIAGNOSIS IN DEPRESSION}

fMRI may also be useful in distinguishing those with unipolar and bipolar depression. Each of these disorders has a different aetiology and the optimal treatments are different. It is difficult to separate the two in a patient presenting solely with depressive symptoms, without a history of bipolar disorder. Estimates are that as many as $28 \%$ of those presenting with only depression will go on to develop bipolar disorder (Hantouche et al., 1998). Functional imaging studies show distinct patterns of activation in the two disorders. Those with bipolar disorder show persistent overactivation in ventromedial prefrontal cortex when depressed, compared with underactivation in unipolar depression (Savitz and Drevets,
2009). fMRI could therefore be a screening tool for those with depression, allowing optimal treatment to be started early in the course of the illness.

The main caveat is that all of these results are from studies on carefully selected research populations, without the normal comorbidities and variability of those seen in everyday clinical practice. Further studies are needed to demonstrate the generalisability of these promising data. Research is also needed to establish the cost benefit of the introduction of fMRI into clinical practice.

\section{CONCLUSION}

MRI is rarely used in clinical psychiatric practice, with a lack of current evidence that it is cost effective in improving diagnosis. There are particular reasons why patients with mental illness may have difficulty in taking part in MRI studies, and there are particular concerns about the implications of structural brain differences on individual experience of illness. However other patients embrace the potential to demonstrate biological differences to explain the subjective experiences of mental illness. Whilst it is difficult to argue the case for routine use of structural MRI for those with mental illness there are exciting possibilities for the use of functional MRI in diagnosing and planning treatment for those with depression, such that the optimal management of those with depression in the near future should include the use of fMRI.

\section{REFERENCES}

Chen, C. H., Suckling, J., Ooi, C., Fu, C. H., Williams, S C., Walsh, N. D., Mitterschiffthaler, M. T., Pich, E. M., and Bullmore, E. (2008). Functional coupling of the amygdala in depressed patients treated with antidepressant medication. Neuropsychopharmacology 33, 1909-1918.

Craddock, N., Antebi, D., Attenburrow, M. J., Bailey, A., Carson, A., Cowen, P., Craddock, B., Eagles, J., Ebmeier, K., Farmer, A., Fazel, S., Ferrier, N., Geddes, J., Goodwin, G., Harrison, P., Hawton, K., Hunter, S. Jacoby, R., Jones, I., Keedwell, P., Kerr, M., Mackin, P., McGuffin, P., Macintyre, D. J., McConville, P., Mountain, D., O’Donovan, M. C., Owen, M. J., Oyebode, F., Phillips, M., Price, J., Shah, P., Smith, D. J., Walters, J., Woodruff, P., Young, A., and Zammit, S. (2008). Wake-up call for British psychiatry. Br. J. Psychiatry 193, 6-9.

Drevets, W. C., Price, J. L., Simpson, J. R. Jr., Todd, R. D., Reich, T., Vannier, M., and Raichle, M. E. (1997) Subgenual prefrontal cortex abnormalities in mood disorders. Nature 386, 824-827.

Fu, C. H., Williams, S. C., Cleare, A. J., Scott, J., Mitterschiffthaler, M. T., Walsh, N. D, Donaldson, C., 
Sucklin, J., Andrew, C., Steiner, H., and Murray, R. M. (2008). Neural responses to sad facial expressions in major depression following cognitive behavioral therapy. Biol. Psychiatry 64, 505-512.

Hantouche, E. G., Akiskal, H. S., Lancrenon, S., Allilaire, J. F., Sechter, D., Azorin, J. M., Bourgeois, M., Fraud, J. P., and Châtenet-Duchêne, L. (1998). Systematic clinical methodology for validating bipolar-II disorder: data in mid-stream from a French national multi-site study (EPIDEP). J. Affect. Disord. $50,163-173$.
Lennox, B. R., Park, S. B. G., Jones, P. B., Medley, I., and Morris, P. G. (2001). A functional anatomy of auditory hallucinations in schizophrenia. Psychiatry Res. Neuroimaging 100, 13-20.

Marneros, A. (2008). Psychiatry's 200th birthday. Br. J. Psychiatry 193, 1-3.

Reil, J. (1808). Beyträge zur Beförderung einer Kurmethode auf psychischem Wege. Halle, Curtsche Buchhandlung.

Savitz, J., and Drevets, W. C. (2009). Bipolar and major depressive disorder: neuroimaging the developmental-degenerative divide. Neurosci. Biobehav. Rev. 33, 699-771.

Received: 15 July 2009; published online: 23 December 2009. Citation: Front. Hum. Neurosci. (2009) 3:46. doi: 10.3389/neuro.09.046.2009

Copyright $\odot 2009$ Lennox. This is an open-access article subject to an exclusive license agreement between the authors and the Frontiers Research Foundation, which permits unrestricted use, distribution, and reproduction in any medium, provided the original authors and source are credited. 